\title{
UN MÉTODO PARA EL TRATAMIENTO INFORMÁTICO DE MATERIALES LATINOS EN VERSO
}

To date, the use of information science applied to Latin versification (automatic scansion methods, various systems for the analysis of texts composed in verse, etc.) has been limited almost exclusively to the study of the hexameter.

In this paper we propose a method for the computerized study of other meters (iambotrochaics, dactylics, anapaestics, eolics), taking into account the specific metrical structure of each one. At the same time, an attempt is made to investigate the possibility of comparing the use of the same lexical elements in different metric forms.

1. Desde hace años la utilización de ordenadores en los estudios de métrica latina ha dejado de ser una promesa esperanzadora para convertirse en una fructifera realidad.

Se han arbitrado métodos de escansión automática, se han diseñado sistemas y códigos para el análisis de textos tanto a nivel de "esquema» como a nivel de "composición $»^{1} \mathrm{y}$, sobre todo, se han aplicado algunos de estos sistemas lo suficiente como para que sea ya un hecho constatado su alto rendimiento ${ }^{2}$.

${ }^{1} \mathrm{Cf}$., por ejemplo, L. Nougaret, «Une méthode de dépouillement destinée aux index métriques", REL 40, 1962, pp. 136-141; los numerosos trabajos de G.E. Duckworth (culminados en su Vergil and classical hexametrical poetry. Ann Arbor 1969) o los de F.P. Jones ("A binary-octal code for analyzing hexameters», TAPhA 97, 1966, pp. 275-280, "Notes on the input for an automatic scansion program", Revue, 1968/4, pp. 5-11), N.A. Greenberg y W. Ott, ligados a las actividades del L.A.S.L.A. de la Universidad de Lieja y a su Revue.

Entre nosotros, además del informe de E. Rodón en RSEL 4, 1974, pp. 488-503, cf. F. Meléndez y Jiménez, "Contribución al análisis de la estructura del hexámetro latino mediante ordenadores electrónicos", RSEL 4, 1974, pp. 230-237.

2 Baste recordar los Materialen zu Metrik und Stilistik de Ott, como valiosos resultados del sistema de análisis por él propuesto (Revue, 1966/4, pp. 7-24, y 1967/1, pp. 39-64) o bien L. Nougaret, Prosodie, métrique et vocabulaire. Analyse verbale com- 
Todos estos trabajos, que por fortuna no son pocos, se han centrado exclusivamente en el hexámetro dactílico, mientras hasta ahora, al menos según nuestra información, se ha desatendido prácticamente todo el resto de la producción latina en verso ${ }^{3}$.

parée du "De Signis» et des "Bucoliques», París 1966, y E. Liénard, Répertoires prosodiques et métriques, Lucrèce, "De rerum natura" L. III etc., Bruselas 1978, en la línea metodológica marcada por Nougaret en «Une méthode...» citado más arriba.

Cf. también J. Perret, "Au service des métriciens un nouvel instrument scientifique", REL 44, 1966, pp. 117-121.

3 Después de estar elaborado el presente trabajo hemos conocido el siguiente código propuesto en su dia por P. Salat («Deux suggestions pour noter la position des mots dans le vers latin", $R E L 47,1969$, pp. 482-491) para los versos yambo-trocaicos, particularmente los de la comedia:

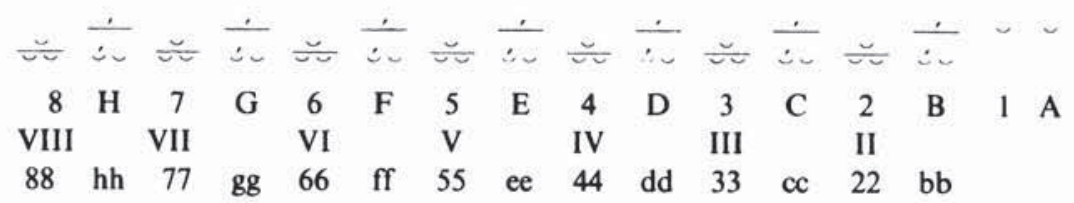

\begin{tabular}{|c|c|}
\hline octonaire & iambique \\
\hline septénaire & trochaïque \\
\hline sénaire & iambique \\
\hline septénaire & iambique \\
\hline
\end{tabular}

Dicho código, deudor según su propio autor del propuesto por Nougaret para el hexámetro y el pentámetro, además de resultar mucho más complejo y, por ello, menos funcional que el que aquí vamos a proponer, adolece de ciertos inconvenientes de diversa gravedad para los objetivos que aquí perseguimos.

Algunas de las dificultades las reconoce el propio autor: por ejemplo (p. $484 \mathrm{~s}$ ), la ambigüedad que lleva consigo el empleo de números romanos junto a números árabes y, sobre todo, junto a caracteres alfabéticos, ambigüedad que, según él, se solucionaría sustituyéndolos por números árabes en negrita. Pero evidentemente tal solución no vale en informática.

Reconoce asimismo (p. 485) los equívocos a que puede dar lugar la caracterización de los tiempos bisilábicos a base de repetir una letra o una cifra, sobre todo cuando cada una de las dos sílabas pertenece a una palabra distinta. La solución que propone a este problema tampoco sería muy eficaz, dado que complicaría aún más el código.

A tales inconvenientes se podrían añadir otros: por ejemplo el tener que recurrir (p. 485) a la inversión de los dos signos finales (1 A) para representar un verso escazonte (A 1); el no distinguir si es larga o breve la silaba anceps final («La syllabe finale est reputée longue, quelle que soit sa quantité»: p. 484); el no tener en cuenta la posibilidad, aunque sólo sea esporádica, de que se encuentre una sílaba breve en lugar de una larga en un tiempo marcado interior; el no distinguir, como hacía Nougaret y como vamos a hacer nosotros, la parte inicial de la parte final del verso; el partir en la notación de tiempos del verso de atrás hacia adelante. 
2. La atención prioritaria al hexámetro en la poesía latina está más que justificada tanto por el volumen de los materiales conservados, como por la propia importancia del verso en sí y por su trascendencia para la lengua poética y, en general, para toda la historia del latín.

La atención exclusiva, en cambio, se comprende, pero no se justifica. Se comprende quizá por su relativa simplicidad de funcionamiento tanto a nivel de esquema como a nivel de composición, sobre todo si se lo compara con otros versos como los yámbicos o trocaicos de la poesía dramática. Ya los antiguos métricos y gramáticos nos muestran un «desequilibrio" semejante, cuando los vemos analizar minuciosamente los esquemas del hexámetro y pasar más o menos sobre ascuas sobre los del trímetro yámbico ${ }^{4}$. En la métrica moderna, en general, la situación no parece haber sido muy distinta. Desde Hultgren ${ }^{5}$ o Birt ${ }^{6}$ hasta Duckworth ${ }^{7}$ se han estudiado con absoluto detenimiento y detalle las particularidades del esquema ${ }^{8} \mathrm{o}$ de la composición del hexámetro, sin que se pueda decir que haya habido un tratamiento parecido o comparable de otros materiales versificatorios.

Se puede comprender, repetimos, semejante discriminación por la particular importancia (e incluso en algunos casos por la mayor simplicidad) del hexámetro. Pero no se justifica el que se dejen a un lado otros versos, como algunos yámbicos o trocaicos o como los eólicos, privándolos de los indudables beneficios que las técnicas informáticas están aportando al estudio del padre hexámetro.

La posible ventaja que podría suponer el identificar cada uno de los tiempos con el mismo número o letra $(8 / \mathrm{VIII} / 88 ; \mathrm{H} / \mathrm{hh} ; 7 / \mathrm{VII} / 77$, etc.) no es tal, si para ello hay que recurrir, como se recurre, a los números romanos $\mathrm{y}$, sobre todo, a la duplicación de un mismo carácter, duplicación que, como ya hemos dicho, conlleva entre otros inconvenientes el de renunciar a caracterizar cada una de las posibles sílabas del verso con un signo distinto.

A todo ello habría que sumar el que un código como éste no se plantea la posible necesidad de ser compatibilizado con el de otro tipo de versos (ni siquiera con el propuesto por Nougaret para el hexámetro y el pentámetro, a pesar de que explícitamente lo toma el autor como modelo) y que, por último, tampoco parece resolver adecuadamente el problema de la enclisis y la elisión (o la aféresis), sobre todo la de las palabras monosilábicas.

${ }^{4}$ Cf., por ejemplo, Sacerdote, GLK VI 502 ss., 517 ss., 528 ss.

5 F.C. Hultgren, "Obseruationes metricae in poetas elegiacos Graecos et Latinos", Programm des Nicolaigymnasiums, Leipzig 1871, pp. 1-37; 1872, pp. 1-34.

6 Th. Birt, Ad historiam hexametri Latini symbola, Bonn 1877.

7 Op. cit. en nota 1.

8 Cf. H. Cancik, H. Cancik-Lindemaier, D. Kottke, W. Ott, «Untersuchungen zur Geschichte der Schemata-forschung in Lateinischen Hexameter", Revue, 1979/3, pp. 1-86. 
Es más, en cierto modo se puede decir que el propio estudio del hexámetro queda incompleto, si los datos que de él se obtienen no cuentan con la posibilidad de ser cotejados con los procedentes de otros ámbitos de la versificación. Piénsese, por ejemplo, en los repertorios prosódicométricos, en los que se estudian y organizan las palabras según su estructura prosódica y su posición en el hexámetro: ¡cuánto material interesante nos ofrecen en este sentido los versos eólicos (dada su peculiar naturaleza silábico-cuantitativa) o los versos yambo-trocaicos! ¡Cuántos datos de interés puede brindar la comparación del empleo de unos mismos materiales léxicos en distintas formas métricas, en versos de ritmo distinto! Mucho más si se tienen en cuenta aquellos versificadores (Horacio, por ejemplo) cuya obra abarca varias formas de versificación.

3. Pues bien, en este sentido va la propuesta que aquí hacemos, una propuesta meramente indicativa y concretada por tal motivo en aquellos versos cuyo estudio puede resultar más rentable tanto por su propia entidad cuanto por la amplitud de materiales conservados: el trímetro y dimetro yámbicos, con sus diversas variantes ${ }^{9}$, el «septenario» trocaico, el dímetro anapéstico (con su variante cataléctica, el "paremíaco»), los versos eólicos.

Centrándonos, por tanto, en estos versos, queremos proponer un sistema que facilite el procesamiento y análisis informático de los materiales en ellos escritos; un conjunto de códigos lo más unívoco posible, de modo que permita relacionar entre sí (y con los del hexámetro) tanto los esquemas métricos de los distintos versos, como, y sobre todo, los elementos de la composición de cada uno de ellos; un método que intenta aunar dos extremos de suma importancia: la eficacia y la simplicidad.

Tomamos como base la propuesta metodológica de Nougaret ${ }^{10}$, lo cual equivale a centrarnos en la tipología verbal. Pero, como vamos a ir viendo enseguida, a partir de dicha base tipológica son otros muchos los elementos no sólo de la composición sino del esquema del verso a que se puede atender.

4. Utilizamos en cada verso un signo distinto para cada uno de los posibles elementos (sílabas largas o breves) de su esquema.

Intentamos que dichos signos empleados en la codificación reflejen en la medida de lo posible la entidad dentro del esquema de los elementos que representan:

a) Así, por ejemplo, distinguimos los elementos iniciales y finales empleando letras, respectivamente, iniciales y finales del alfabeto.

\footnotetext{
9 A los que se pueden añadir el octonario y el septenario.
}

10 "Une méthode...», citado en la nota 1. 
b) Con la excepción, por las razones que se indicarán, del «septenario" trocaico, mantenemos la $\mathrm{Z} / \mathrm{z}$ como signo de la sílaba final de los versos acatalécticos y la $\mathrm{Y} / \mathrm{y}$ de la de los versos catalécticos.

c) En los versos cuantitativos (versos de pies) distinguimos entre tiem$\operatorname{pos}^{11}$ monosilábicos largos (representándolos siempre por mayúsculas), tiempos monosilábicos breves (minúsculas) y tiempos disilábicos (un número para cada una de sus sílabas ${ }^{12}$ ).

d) En los versos silábico-cuantitativos (eólicos) utilizamos mayúsculas para las sílabas largas, minúsculas para las breves aisladas y números para los grupos de dos breves («coriambo»: 12 para el primero, 34 para el segundo, etc.).

Además de estos signos alfabéticos y numéricos (y del eventual recurso a algún otro signo del código ASCII) utilizamos, de la manera que se explicará más adelante, los siguientes: «(»= elisión; «)» = aféresis; «*» =enclisis; «\#» = enclisis + elisión; «—》 = hiato.

La mayor complejidad del esquema de algunos de los versos que vamos a analizar no permite en este sistema distinguir, como hacía Nougaret con el hexámetro y el pentámetro, cada pie (con una letra) y sus tiempos marcado (mayúscula) y.no marcado (minúscula) ${ }^{13}$. Hay, por tanto, en nuestros códigos cierta inevitable incongruencia con los del maestro francés. Puede que en alguna ocasión se hiciera necesario compatibilizarlos por completo (por ejemplo, para analizar materiales de un poeta que escribe en hexámetros y en otros versos de los que aquí estudiamos): en tal

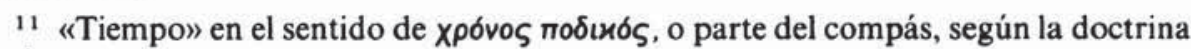
rítmica.

${ }_{12}$ Un número en la medida de lo posible, pues a partir de una determinada longitud del verso no bastan los diez signos $\varnothing-9$; se recurre entonces, según veremos, a las letras minúsculas no empleadas para los tiempos breves (generalmente las de la parte central del alfabeto) e incluso a veces habrá que echar mano de otros signos del código ASCII.

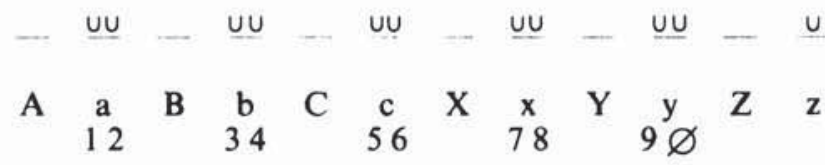

Un sistema así puede resultar más «visual» para un tratamiento «a mano» de los materiales; ahora bien a la hora de procesar y tratar informáticamente dichos materiales no añade rentabilidad: lo mismo da el pie primero sea $A_{12}^{a}$ que $A \underset{12}{B}$. Por otra parte, este sistema de Nougaret, como el que antes veíamos de Salat, no permite distinguir (no era rentable en el hexámetro, pero sí lo es en algunos otros versos, por ejemplo, para determinar la existencia o no de synaphia) si al final se emplea sílaba larga o breve. 
caso es fácil traducir a nuestro código el de Nougaret a base de analizar el hexámetro

$$
\begin{aligned}
& \begin{array}{llllllllllll}
\text { A } & \text { B } & \text { C } & \text { D } & \text { E } & \text { F } & \text { U } & \text { V } & \text { W } & \text { X } & \text { Y } & \text { Z }
\end{array} \\
& \begin{array}{llllll}
12 & 34 & 56 & 78 & 9 \varnothing & \mathrm{z}
\end{array}
\end{aligned}
$$

y el pentámetro

$$
\begin{aligned}
& \begin{array}{llllllllll}
A & B & \text { C } & \text { D } & \text { E } & \text { V } & \text { W } & \text { X } & \text { Y } & \text { Z }
\end{array} \\
& \begin{array}{lllll}
12 & 34 & 78 & 9 \varnothing & \mathrm{Z}^{14}
\end{array}
\end{aligned}
$$

Una vez trazadas las líneas generales de nuestro sistema, procede ir concretando y descender a nivel de un verso concreto. Lo vamos a hacer con el trímetro/senario yámbico.

1. Proponemos para este verso el siguiente código

$$
\begin{array}{cccccccccccc}
\text { a } & & \text { c } & & \text { e } & & \text { u } & & \text { w } & & \text { y } & \text { Z } \\
\text { A } & \text { B } & \text { C } & \text { D } & \text { E } & \text { T } & \text { U } & \text { V } & \text { W } & \text { X } & \text { Y } & \text { Z } \\
12 & 34 & 56 & 78 & \text { hi } & \text { jk } & \text { Im } & \text { no } & \text { pq } & \text { rs } & &
\end{array}
$$

En los tiempos monosilábicos distinguimos el primer hemistiquio (ordinariamente penthemimeres) con las primeras letras del alfabeto (A-E) y el segundo, con las últimas (T-Z). Señalamos con minúsculas aquellos tiempos monosilábicos que con más frecuencia pueden ser breves. Por supuesto que, en caso de que accidentalmente («alargamiento en arsis», indiferencia ocasional del elemento) cualquiera de los otros elementos (B, D, T, V, X) sea breve, se deberá emplear la minúscula correspondiente.

Para los tiempos bisilábicos empleamos ocho dígitos (1-8) y doce letras minúsculas (h-s), aprovechando la necesidad de combinar signos numéricos y alfabéticos para distinguir también aquí el primer hemistiquio del segundo ${ }^{15}$.

14 Obsérvese, por ejemplo, una primera coherencia que se consigue con esta nueva codificación: la cesura (o colon) penthemímeres queda caracterizada por la letra $\mathrm{E}$, coincidiendo tal caracterización con la que enseguida veremos en otros versos, como, por ejemplo, el trímetro yámbico.

15 La mezcla de signos numéricos y alfabéticos responde al deseo de buscar una codificación de los tiempos bisilábicos que permita distinguir cualquiera de los elementos del verso de todos los demás y a la vez ubicarlo en el esquema general del verso.

Evidentemente podría presentarse el caso de que para un estudio concreto resultase más rentable destacar la posición de cada elemento dentro de cada metro, sin necesidad de caracterizarlo frente a todos los demás elementos del verso; en tal caso la codificación más útil sería

$\begin{array}{llllllllll}12 & 34 & 56 & 78 & 12 & 34 & 56 & 78 & 12 & 34 .\end{array}$


Este sistema permite analizar cualquier trimetro o senario por muy complejo que sea y obtener a partir de dicho análisis una variada gama de datos tanto sobre los esquemas como sobre la composición de dichos esquemas.

2. Baste como ejemplo el análisis de los siguientes versos plautinos y terencianos ${ }^{16}$ :

80 quasi cum caletur cocleae in occulto latent

$$
12 \quad|\mathrm{~B} \quad| \mathrm{cDE} \quad \text { ljk } \quad|\mathrm{u} \quad| \mathrm{VWX} \quad \mid \mathrm{yZ}
$$

81 suo sibi suco uiuunt, ros si non cadit:

$$
\begin{array}{llllllll}
\mathrm{aB} & 156 & \mid \mathrm{DE} & \mid \mathrm{TU} & \text { IV } & \text { IW } & \text { |X } & \text { |yZ }
\end{array}
$$

82 item parasiti rebus prolatis latent

aB $\quad|56 \mathrm{DE}| \mathrm{TU} \quad|\mathrm{VWX} \quad| \mathrm{yZ}$

83 in occulto miseri, uictitant suco suo,

$1 \quad|2 B C \quad| 78 \mathrm{E} \quad|\mathrm{TuV} \quad| \mathrm{WX} \quad \mid \mathrm{yZ}$

84 dum ruri rurant homines quos ligurriant.
A
$\mid \mathrm{BC}$
|DE
ljkU |V
|wXyZ

85 Prolatis rebus parasiti uenatici

$\mathrm{ABC} \quad$ IDE |jkUV |WXyZ

86 sumus: quando res redierunt, molossici

$$
\text { aB } \quad|C D \quad| E \quad \text { ljkUV } \quad \text { wXyZ }
$$

87 odiosicique et multum incommodestici.
$12 \mathrm{BcD} \#$ \# \#
IE $\quad \mid \mathrm{T}$
|UVwXyZ

88 Et hic quidem hercle, nisi qui colaphos perpeti
|B $\quad$ |c $\quad$ |DE ljk |U
InoW $\quad \mid X y Z$

a

89 potis parasitus frangique aulas in caput aB |56DE |TU\# I\# |VW |X |yZ

384 uidere uideor iam diem illum, cum hinc egens $\begin{array}{llllllll}\mathrm{aBc} & 178 \mathrm{E} & \mid \mathrm{T} & \text { |u } & \mid \mathrm{VW} & \mid \mathrm{I} & \mid \mathrm{X} & \mid \mathrm{yZ}\end{array}$

783 men criminatust? optumest: ego sum malus $\begin{array}{lllllll}A \# \quad|\# \quad| \mathrm{BcDE})|| & \mid \mathrm{TuV}) \quad \mid) \quad \mid \mathrm{pq} & \mid \mathrm{X} & \mid \mathrm{yZ}\end{array}$

124 ne se appellarem; deinde, postquam ocassio est
A $\left.\quad\right|^{*} \quad \mid$ BCDE $|\mathrm{Tu} \quad| \mathrm{V}(\quad \mid$ WXyZ $\left.) \mid\right)$

821 nec sentit, tantist quantist fungus putidus
A $\quad \mid B C$
(DE) |)
(TU) ()
IVW $\quad$ XYZ

392 pudet pigetque. Nimium inter uos, Demea aB $\quad\left|\mathrm{CDe}^{*}\right|^{*} \quad$ ljk $\quad|\mathrm{uV} \quad| \mathrm{W} \quad \mid \mathrm{XyZ}$ y 392 .

16 Plauto, Captiui 80-89, Bacchides 783 y 821, Miles 124; Terencio, Adelphoe 384 
3. Si lo que se pretende es únicamente almacenar y procesar el léxico (con la indicación en cada palabra de su estructura prosódica y su posición en el verso), basta con fichar el material palabra por palabra añadiéndole a cada una la fórmula correspondiente.

En cambio, si el análisis se enfoca desde una perspectiva más ambiciosa, con el deseo de estudiar este o aquel aspecto del esquema o de la composición de cada uno de los versos, se deberá trabajar sobre una hoja de datos adecuadamente organizada, determinando previamente el campo a asignar a cada una de las posibles palabras de los versos. Por ejemplo, contando con un máximo de diez palabras por verso y de ocho sílabas por palabra se podría distribuir una línea de ochenta casillas en diez campos (uno por cada palabra) de ocho casillas cada uno: $1 .^{\mathrm{a}}$ palabra, casillas $1-8 ; 2$. $^{a}$ palabra, casillas $9-16 ; 3 .^{a}$ palabra, casillas $17-24$, etc. En cada campo, empezando por el primero, se describiría el esquema prosódico de la palabra en cuestión según el código antes descrito.

4. Atención especial merecen fenómenos como la elisión, la aféresis, la enclisis o el hiato.

En caso de elisión, si se trata de palabras polisílabas, se añade el signo «(» a la fórmula de la palabra correspondiente. Por ejemplo, Capt. $80 \mathrm{co}$ cleae $=\mathrm{jk}(; 88$ quidem $=\mathrm{c}$.

Si la palabra elidida es un monosílabo, se colocará únicamente el signo "(», dejando el resto del campo en blanco:

Adelph. 384 uidere uideor iam diem illum, cum hinc egens

$$
\begin{array}{lllllll}
\mathrm{aBc} & 78 \mathrm{E} & \mathrm{T} & \mathrm{u} & \mathrm{VW} & (\mathrm{X} & \mathrm{yZ}
\end{array}
$$

La aféresis que tiene lugar con est y es se representa añadiendo el signo (1)" a la fórmula de la palabra en que se apoyan tales formas verbales y volviendo a escribir dicho signo en la primera casilla del campo siguiente, es decir, del campo que les habría correspondido a estas palabras de no haber sufrido aféresis. Véanse en este sentido los textos antes citados de Bacch. 783 y 821 y Miles 124 .

Las enclíticas -que, -ne, -ue se cuentan formando una sola unidad prosódica con la palabra en que van apoyadas. Su presencia se indicará colocando, tanto al final de la fórmula de la palabra anterior como en la primera casilla del campo que, de ser palabra autónoma, le habría correspondido, el signo *; el resto de dicho campo quedará en blanco. Véase, por ejemplo, Adelph. 392.

En caso de que la enclítica sufra a su vez elisión, se indicará de la misma forma, pero con el signo "\#». Por ejemplo, Captiui 87, 89, Bacch. 783.

El hiato se representa añadiendo el signo «- a la fórmula de la primera de las dos palabras. 
Si el despojo de los textos no se hace por versos, sino por palabras, basta añadir cada uno de estos signos a la fórmula prosódica correspondiente. En consecuencia, en los casos de monosílabos o enclíticos elididos sólo figurarán en dicha fórmula los signos «(» $\mathrm{o}$ «\#».

5. A partir de un texto despojado y preparado por un procedimiento como el que acabamos de describir se puede conseguir un índice de palabras en el que cada una figure con su(s) fórmula(s) métrico-prosódicas (es decir con la indicación tanto de su estructura cuantitativa cuanto de la(s) posición(es) que ocupa en el verso).

E igualmente, una variada serie de listados y recuentos, como, por ejemplo, de todas las palabras que se dan bajo una misma «fórmula", del tipo de los que sobre material hexamétrico ofrecen Nougaret y Liénard en sus obras antes citadas.

6. Pero además, con este procedimiento de recogida y procesamiento de materiales se abre una riquísima gama de posibilidades para estudiar el senario/trímetro yámbico tanto a nivel de esquema como a nivel de composición y para poder verificar a través de semejante estudio los rasgos peculiares de un autor, un género (comedia/fábula/tragedia/lírica), una época, etc.

A nivel de esquema (además de que el ordenador puede reproducir el de cada verso con los signos tradicionales ( $u /-$ ) simplemente con la orden de reescribir por "u» todos los números y minúsculas y por «-" todas las mayúsculas) las posibilidades van desde lo que pueden ser simples recuentos y estudios de frecuencias, hasta la determinación del grado en que el material procesado se aproxima al "senario» o al «trímetro»; el funcionamiento de las variedades "puro» o "escazonte»; el grado de resolución de cada uno de los dos tiempos de los distintos pies; el recurso o no a la sílaba anceps en final de verso o en otros lugares como los anteriores a pausa o los loci Iacobsohniani ${ }^{17}$; el estudio del tratamiento de las series de cuatro breves seguidas, por ejemplo, en lo que se refiere a la aceptación del proceleusmático y al rechazo de la secuencia tríbraco o dáctilo más anapesto, rasgo éste típico de los versos yámbicos frente a los trocaicos ${ }^{18}$.

Y si esto es así a nivel de esquema, en la composición resulta aún más rentable el procedimiento que estamos proponiendo, sobre todo si se tiene

17 C. Questa, Introduzione alla metrica di Plauto, Bolonia 1967, pp. 146 y 151 ss.

18 Cf., por ejemplo, D.S. Raven, Latin Metre, Londres 1965, p. 45. Según esto, se mostrarán en el trímetro yámbico las secuencias 1234,5678 , hijk, lmno, pqrs (= proceleusmático) más frecuentes que las $3456,78 \mathrm{hi}, \mathrm{jklm}$, nopq ( $=$ tríbraco o dáctilo más anapesto). 
en cuenta lo complejo y variado de los elementos que entran en juego a este nivel ${ }^{19}$.

Limitándonos a los datos introducidos anteriormente (forma prosódica de cada una de las palabras del verso ${ }^{20}$ ), se puede estudiar en primer lugar el funcionamiento de las cesuras y zeugmas ${ }^{21}$. Y a partir de aquí se pueden llevar a cabo todo tipo de precisiones sobre la tipología verbal y se pueden verificar tantas y tantas observaciones como se han hecho por parte de los metricólogos modernos sobre el senario de la comedia enfocando unas veces dicha tipología en función del esquema ${ }^{22}$ o estudiándola en otras ocasiones en relación (positiva o negativa) con la posible búsqueda consciente de la homodinia por parte de los versificadores ${ }^{23}$.

Es viable incluso un estudio indirecto de la homodinia/heterodinia realizado a través de la tipología verbal. Téngase en cuenta, por ejemplo, que B, D, T, V, X, si son iniciales de bisílabos, entrañan homodinia; si son finales de bisilabos, heterodinia (excepto si van seguidos de «(»). En cambio, A/a, C/c, E/e, U/u, W/w, si son penúltimas en un conjunto bisilábico, conllevan heterodinia. En las unidades trisilábicas B, D, T, U, X, penúltimas o ante «(", indican homodinia; asimismo c, e, u, w, y, suponen un acento secundario en la sílaba siguiente, si son penúltimas.

A todo lo anterior se pueden añadir estudios sobre el número de sílabas de los versos, de los hemistiquios o de las palabras; sobre el número de palabras de los versos; sobre la relación palabra/pie; sobre la elisión, la enclisis, el hiato, etc.

19 J. Luque, «Niveles de análisis en el lenguaje versificado», Athlon. Satura grammatica in honorem Francisci $R$. Adrados, vol. I, Madrid 1984, pp. 287-299, especialmente $291 \mathrm{~s}$.

20 A ellos se podrian haber añadido otros como la clase o la función de las palabras, por un procedimiento similar al que hemos utilizado en otra ocasión: J. Luque, "Un modelo de tratamiento de textos latinos en hexámetros», Estudios de Filología Latina IV, Granada 1985 (en prensa).

${ }_{21}$ Basta, por ejemplo, un recuento de las palabras terminadas en $\mathrm{E}(\mathrm{e}, \mathrm{hi})$ o U (u, Im) para determinar la frecuencia, respectivamente, de la penthemimeres o hepthemimeres. Basta igualmente, para ver si se respeta o no el zeugma de Porson, comprobar la presencia de las palabras X Y Z Z precedidas de otras terminadas en W.

${ }_{22}$ Véase, por ejemplo, L. Nougaret, Traité de Métrique latine classique, París 1963, p. 71 ss. Según ello serán frecuentes las palabras (no monosilábicas) o fines de palabra en $\mathrm{cD}, \mathrm{c78}, \mathrm{uV}$, uno, AB, A34, ET, EjK, WX, Wrs.

${ }_{23}$ En este sentido véanse, por ejemplo, Raven, op. cit., p. 55 ss. o Questa, op. cit., p. 124 ss., donde se recoge gran parte de los estudios clásicos en este campo. Así, por ejemplo, según las «normas» de Ritschl o de Hermann-Lachmann quedarán muy restringidos los fines de palabra en $5,6,7,8, \mathrm{~h}, \mathrm{i}, \mathrm{j}$, etc.; según la norma de FraenkelThierfelder-Skutsch, si hay fin de palabra en 1, 5, h, l, p, no pueden aparecer, respectivamente, 34,78 , jk, no, rs. 
La información que a partir de todo ello se puede obtener resulta de gran valor para el estudio del trímetro/senario yámbico, tanto en un sentido diacrónico a través de autores y épocas, como en un sentido sincrónico, precisando los rasgos característicos de sus distintas especies (puro, trímetro, senario, escazonte) y a través de ello la caracterización de los géneros literarios que los emplean.

Todo cuanto con cierto detalle acabamos de plantear para el trimetro yámbico se puede aplicar mutatis mutandis a otros versos.

1. Sin salir de los de ritmo yámbico, el tetrámetro/octonario o el «septenario" no of recerian más dificultad que el elevado número de elementos de tiempos resueltos, número que obligaría a utilizar en la codificación además de minúsculas y números, algunos otros signos.

Una fórmula podría ser ésta:

$$
\begin{array}{lllllllllllllll}
\text { a } & & \text { c } & & \text { e } & & \text { g } & \text { h } & \text { s } & & \text { u } & & \text { w } & & \text { y } \\
\text { A } & \text { B } & \text { C } & \text { D } & \text { E } & \text { F } & \text { G } & \text { H } & \text { S } & \text { T } & \text { U } & \text { V } & \text { W } & \text { X } & \text { Y } \\
12 & 34 & 56 & 78 & 9 \varnothing & \text { ij } & , . & : ; & \text { kl } & \text { mn } & \text { op } & \text { qr } & <=>/
\end{array}
$$

Los versos más cortos que el trímetro no presentan problema alguno. Para cualquiera de ellos se puede proponer una fórmula creada a partir de la que veíamos antes. He aquí algunos a título de ejemplo:

Trímetro cataléctico

$$
\begin{array}{lllllllllll}
\text { a } & & \text { c } & & \text { e } & & \text { u } & & \text { w } & & \text { y } \\
\text { A } & \text { B } & \text { C } & \text { D } & \text { E } & \text { T } & \text { U } & \text { V } & \text { W } & \text { X } & \text { Y } \\
12 & 34 & 56 & 78 & \text { hi } & \text { jk } & \text { lm } & \text { no } & \text { (pq } & \text { rs) } &
\end{array}
$$

Dímetro (cataléctico)

$$
\begin{array}{lllllll}
\text { a } & & \text { c } & & \text { w } & & \text { y } \\
\text { A } & \text { B } & \text { C } & \text { D } & \text { W } & \text { X } & \text { Y } \\
12 & 34 & 56 & 78 & \text { pq } & \text { rs } &
\end{array}
$$

\footnotetext{
${ }^{24}$ El empleo de otros signos se entiende necesario en el supuesto de que se necesite distinguir cada uno de los elementos en cuestión de los demás del mismo verso. Evidentemente, si sólo se necesita distinguirlos por metros, basta, según vimos más arriba, con enumerarlos del 1 al 8 dentro de cada metro.

El reservar en la fórmula que presentamos los pies cuarto y séptimo para estos otros signos obedece a que es en dichos lugares donde posiblemente aparecerán con menos frecuencia. Cabe, por supuesto, cualquier otra ordenación, aunque en tal caso la más conveniente sería la que tuviese en cuenta los valores de todos los caracteres empleados (números, letras y otros signos) dentro del código ASCII.
} 
2. Para el «septenario» trocaico se podría proponer el siguiente código:

$$
\begin{array}{lllllllllllllll} 
& \text { b } & & \text { d } & & \text { f } & & \text { h } & & \text { u } & & \text { w } & & \text { y } & \text { Z } \\
\text { A } & \text { B } & \text { C } & \text { D } & \text { E } & \text { F } & \text { G } & \text { H } & \text { T } & \text { U } & \text { V } & \text { W } & \text { X } & \text { Y } & \text { Z } \\
\text {,. } & : ; & 9 \varnothing & 12 & 34 & 56 & 78 & & \text { jk } & \text { lm } & \text { no } & \text { pq } & \text { rs } & &
\end{array}
$$

En la asignación de caracteres a los distintos elementos procuramos mantener aquí cierta correspondencia con la fórmula del trímetro yámbico, reflejando así de algún modo la relación entre la forma rítmico-métrica de estos dos versos:

$$
\begin{array}{r}
-u-u-u-u+-u-u-u \underline{u} \\
u-u-u \quad / \quad-u-u-u \underline{u}
\end{array}
$$

Por buscar esta correspondencia parece preferible mantener aquí una fórmula terminada en $\mathrm{Z} / \mathrm{z}$, a pesar de tratarse de un verso cataléctico. De todos modos, dicha "anomalía» no es tan estridente como podría resultarlo en un caso en que la variante cataléctica de un verso tuviera igual o superior importancia que la cataléctica, caso que, evidentemente, no es el del «septenario» trocaico, con cuyo amplio desarrollo en la poesía latina no se puede comparar el reducido empleo del tetrámetro/octonario trocaico acataléctico.

3. Dentro de los versos anapésticos, para unas formas tan comunes como el dímetro o su variante cataléctica (paroemiacus) se podría usar una fórmula como ésta:

$$
\begin{array}{lllllll}
\text { A } & \text { B } & \text { C } & \text { D } & \text { W } & \text { X } & \text { Y } \\
12 & 34 & 56 & 78 & \text { ef } & \text { gh } & \text { ij }
\end{array}
$$

La versificación eólica pide, en cambio, un tratamiento especial que recoja, por una parte, sus peculiaridades estructurales y que garantice la mayor rentabilidad posible en el tratamiento de los materiales compuestos a base de dichos versos: interesa en este sentido establecer unos tipos de fórmulas que reflejen las posibles concomitancias formales entre unos versos y otros y que, gracias a ello, permitan luego interrelacionar los materiales lingüísticos analizados. Tomamos para ello como base la descripción de los esquemas élicos adoptada por nosotros en otra ocasión ${ }^{25}$.

25 J. Luque Moreno, «Las formas eólicas en la métrica latina: propuesta de análisis", Cuadernos de filología clásica (en prensa). 
1. Los principios básicos con que han sido diseñadas las fórmulas que vamos a presentar son los siguientes:

$\left.1 .^{\circ}\right)$ Distinguir, como siempre, los comienzos (primeras letras del alfabeto) de los finales de verso (letras últimas).

$\left.2 .^{\circ}\right)$ Reflejar en cierto modo el principio de isosilabia y, sobre todo, los restos de la originaria indiferencia cuantitativa que aún mantienen los versos eólicos latinos, a base de una letra (mayúscula o minúscula, según los casos) para cada sílaba.

$\left.3 .^{\circ}\right)$ Destacar las dos breves del "coriambo» utilizando para ellas números en vez de letras: 12 para el primer (o único) grupo u v; 34 , para el segundo; 56 , para el tercero.

$\left.4 .^{\circ}\right)$ Caracterizar en la codificación las tres secciones que se pueden reconocer en el esquema de estos versos (el comienzo, la parte central y la cadencia final ${ }^{26}$ ), tratando de reflejar a través de ello la relación de unos con otros:

A. En cuanto al «comienzo» distinguimos los siguientes tipos:

Tipo a: con componente inicial $\varnothing$ : Adonio, aristofanio, alcaico decasílabo.

Tipo b: con componente inicial «o o» («base» eolia)

$$
\begin{array}{ll}
\text { Ferecracio, falecio, gliconio, } & \text { a b } \\
\text { asclepiadeo, asclepiadeo «mayor» } & \text { A B }
\end{array}
$$

Tipo c: con componente inicial «- $-\cup \longrightarrow$

$$
\text { Sáfico endecasílabo, Sáfic. «mayor» : A b C D }
$$

Tipo d: con componente inicial « $\underline{u}-u-\underline{u}$ »

$$
\text { Alcaicos eneasílabo y decasílabo : A B c D E }
$$

B. En lo que respecta a la parte central, hemos procurado también la uniformidad de su representación en los distintos versos. De este modo, $\mathrm{F}$ indica siempre primera larga del primer "coriambo" del verso que, a su vez, va «encadenado» (es decir, su segunda larga coincide con la primera sílaba de) con la cadencia: adonio, ferecracio, aristofanio, sáfico endecasílabo, gliconio y alcaico endecasílabo.

\footnotetext{
$26 \mathrm{Cf}$., por ejemplo, G.B. Pighi, "La metrica latina» en La lingua latina nei mezzi della sua espressione. Turin 1968, p. $458 \mathrm{~s}$.
} 
G $\mathrm{H}$ representan siempre las largas del primer «coriambo» del verso, pero "autónomo", es decir, no encadenado con otro "coriambo" o con otras partes del verso: sáfico mayor, asclepiadeo y asclepiadeo mayor.

I indica primera larga de un primer «coriambo» del verso, cuya segunda larga es del tipo J: alcaico decasílabo.

$\mathbf{J}$ indica segunda larga de un primer "coriambo", la cual además es la primera sílaba larga de un segundo "coriambo» (formando, si se prefiere, una secuencia «dactílica») del tipo $U$.

L M representan las largas de un «coriambo» central autónomo como el del asclepiadeo «mayor».

$\mathrm{U}$ indica primera larga de un "coriambo", que no es el primero del verso, cuya última sílaba es, a su vez, la inicial de la cadencia: sáfico «mayor», asclepiadeo, asclepiadeo "mayor».

C. Las cadencias que distinguimos son de dos tipos, uno de ellos con tres variantes:

\begin{tabular}{|c|c|c|c|c|}
\hline & & & $\mathrm{z}$ & \\
\hline Tipo a & $:-u \underline{u}$ & $=$ & X y Z: & $\begin{array}{l}\text { glic., ascl., ascl. «may.», } \\
\text { alc. endec. }\end{array}$ \\
\hline Tipo b.1. & $:-\underline{u}$ & $=$ & $\begin{array}{r}\mathrm{y} \\
X Y: \\
y\end{array}$ & adonio, ferecracio \\
\hline
\end{tabular}

Tipo b.2. $\quad:-u-u=\quad \mathrm{V} w \mathrm{XY}$ : aristofanio, sáf. end., sáf. «may.», alc. decas., alc. endecas.

$\mathrm{y}$

Tipo b.3. $:-u-u-\underline{u}=$ St Vw X Y: falecio

5. $\left.{ }^{\circ}\right)$ Para los casos esporádicos en que algunos poetas latinos ${ }^{27}$ han sustituido el grupo $u$ u por una sílaba larga hemos destinado la letra $\mathrm{K}$.

Para la representación de la eventual resolución de una sílaba larga por dos breves ${ }^{28}$ reservamos las cifras 89 .

2. Según todos estos principios, he aquí las fórmulas que proponemos para los versos eólicos latinos:

\begin{tabular}{|c|c|c|}
\hline Adonio: $-v \cup-\underline{u}$ & $=$ & $\mathrm{F} 12 \mathrm{X} \mathrm{Y}^{\mathrm{y}}$ \\
\hline Ferecracio: $\stackrel{u}{u}-u \cup u$ & $=$ & $\begin{array}{ll}\text { a } & \text { b } \\
\text { A } & \text { B F } 12 X \\
\text { Y }\end{array}$ \\
\hline Aristofanio: $-u \cup-u-u$ & - & F $12 \mathrm{VwX}$ \\
\hline
\end{tabular}

${ }^{27}$ Cf., por ejemplo, Raven, op. cit., p. 138 ss.; Pighi, op. cit., pp. 425 ss. y 492.

28 Cf., por ejemplo, Raven, loc. cit. 
Falecio:

$\underline{u} \underline{u}-u v-u-u-\underline{u}$

Sáfico endecasílabo:

$$
-u-\underline{u}-u v-u-\underline{u}
$$

Sáfico «mayor»:

$$
=\stackrel{a}{\mathrm{~A}} \stackrel{\mathrm{b}}{\mathrm{B}} \mathrm{F} 12 \mathrm{St} \mathrm{Vw} \text { X } \stackrel{\mathrm{y}}{\mathrm{Y}}
$$

(K)

$$
=A \text { bC } \stackrel{d}{D} F 12 V w X \stackrel{y}{Y}
$$

Alcaico decasílabo:

$$
-u v-u v-u-\underline{u} \quad=112 \mathrm{~J} 34 \mathrm{~V} \text { XXY }
$$

Alcaico eneasilabo:

$$
\underline{u}-u-\underline{u}-u-\underline{u}
$$

Gliconio: $\underline{u} \underline{u}-u v-u \underline{u}$

Asclepiadeo:

$$
\underline{u} u-u v-u v-u \underline{u}
$$

Asclepiadeo «mayor»:

$$
\underline{u} \underline{u}-u \text { u }-u
$$

Alcaico endecasílabo:

$$
\underline{u}-u-\underline{u}-u v-u \underline{u}
$$

3. Cotejando todas estas fórmulas se hace patente la relación estructural entre los esquemas de los distintos versos, relación que justifica o explica el funcionamiento de muchos de estos cola o versos en la composición de los poemas.

Véase en este sentido la siguiente tabla:

Adonio

Ferecracio

Aristofanio

Sáfico endecasílabo

Gliconio

Alcaico endecasílabo

Sáfico «mayor»

Asclepiadeo

Asclepiadeo "mayor»

Falecio

Alcaico decasílabo

Alcaico eneasílabo

\section{COMIENZO PARTE CENTRAL CADENCIA}

$\varnothing$
b
$\varnothing$
c
b
d
c
b
b
b
$\varnothing$
$d$

$\mathrm{F}$

$\mathrm{F}$

$\mathrm{F}$

$\mathrm{F}$

$\mathrm{F}$

F

G H

G H

G H LM U

b. 1 .

b.1.

b. 2 .

b.2.

a

a

b.2.

$\begin{array}{cc}\text { I J } & \text { b. } 3.2 \\ \varnothing & \text { b. } .\end{array}$


Como se puede comprobar, todos los versos eólicos empleados en latín, a excepción del alcaico eneasílabo, tienen al menos un coriambo «encadenado" con la cadencia.

Hay tres parejas en estos versos que sólo se diferencian por su comienzo: adonio/ferecracio, aristofanio/sáfico endecasílabo, gliconio/alcaico endecasílabo.

Sólo se distinguen por la cadencia adonio/aristofanio y gliconio/falecio/ferecracio.

Otros se oponen entre sí por la parte central (interpretables los unos como «expansiones» de los otros):

$$
\begin{aligned}
& \text { gliconio/asclepiadeo/asclepiadeo «mayor» } \\
& \text { sáfico endecas./sáfico «mayor» } \\
& \text { aristofanio/alcaico decasílabo. }
\end{aligned}
$$

Se destacan como casos únicos el falecio (único esquema con cadencia b.3.), el alcaico decasílabo (único esquema con "expansión dactílica») y el alcaico eneasílabo (único esquema con «coriambo» $\varnothing$, o sea, sin "parte central».

4. Pero para los fines que aquí perseguimos la uniformidad de las fórmulas que acabamos de proponer no interesa sólo porque ponga más o menos de manifiesto la relación estructural entre los distintos versos, sino y sobre todo, porque, al reflejarla, y al utilizar los mismos signos para elementos estructurales que son los mismos en más de un verso, permitirá luego tratar conjuntamente el material léxico que se haya ido despojando de la(s) obra(s) de un(os) autor(es) escrita(s) en versos eólicos distintos ${ }^{29}$.

\section{J. LUQUe MORENO}

\footnotetext{
29 El presente trabajo guarda relación con el proyecto de investigación "La doctrina métrica de los romanos" que figura con el n. ${ }^{\circ} 0402$ dentro de los planes de la Comisión Asesora de Investigación Científica y Técnica del Ministerio de Educación y Ciencia.
} 